\title{
AN IRSN CONTRIBUTION TO THE UAM PROJECT: THERMAL- HYDRAULIC AND NEUTRONIC UNCERTAINTIES PROPAGATION IN A ROD EJECTION, FIRST RESULTS
}

\author{
A. Sargeni ${ }^{*}$, E. Ivanov $^{1}$ \\ ${ }^{1}$ IRSN \\ BP 17 - 92260 Fontenay-aux-Roses Cedex - France \\ antonio.sargeni@,irsn.fr, evgeny.ivanov@irsn.fr
}

\begin{abstract}
The paper presents our first results of the exercise III-I-2c from the OECD-NEA UAM-LWR benchmark intended to an elaboration of the methodology of uncertainty propagation. The considered case studied a full PWR core behavior in fast $(\sim 0.1 \mathrm{sec})$ rod ejection transient. According to the benchmark, the core represented a Hot Zero Power state. Authors used brute-force sampling propagating nuclear data and thermo-fluid uncertainties using 3D computational IRSN chain HEMERA. It couples the reactor physics code CRONOS and thermal-hydraulic core code FLICA4. The nuclear data uncertainties were represented in a form of cross sections standard deviations (in percentage of the mean cross sections values) supplied by the UAM team. In addition to the original benchmark, the study includes a case with an increased power peak by supplementary rod ejection, i.e. with higher reactivity. Both the results are similar to what we obtained in the mini-core rod ejection: the power standard deviation follows, in percentage of the mean power, the mean power curve. We split the variance with a direct calculation: once the cross sections are modified and the thermalhydraulics inputs are kept constant, another time the contrary. The results show that uncertainties dues to nuclear data dominate over ones due to the thermal-flow area. Furthermore, the major contributors in peak-of-power variance lie in a fast group of cross sections.
\end{abstract}

KEYWORDS: rod ejection, UAM, uncertainties

\section{INTRODUCTION}

The stringent and confident characterization of the accuracy of modern best-estimate simulations tools is, for a long time, of a particular interest expressed by the international, scientific and engineering communities. This interest concerns the design and safety requirements imposed on existing and advanced nuclear reactors.[1]. This is why the OECD-NEA Nuclear Science Committee (NSC) auspices an establishment of an 'Expert Group' (EG) on 'Uncertainty Analysis Modelling' (UAM). Ten years ago the 'Expert Group on Uncertainty Analysis Modelling' (EGUAM) has been created within one of the NSC 'Working Party on Scientific Issuer on Reactor Systems' (WPRS). The EGUAM was aimed to establish and propagate simulation uncertainties through all phases of Light Water Reactor (LWR) design studies launching relevant UAM-LWR benchmark. UAM-LWR was comprised on the following steps: 1) subdivision of the systems/scenarios in a number of steps (exercises); 2) clear identification of the inputs, outputs and the hypotheses for each step; 3) evaluation of uncertainties for each step and 4) propagation of the uncertainties along the entire design studies and through all scenario. 
This UAM-LWR $[2,3,4]$ approach has been translated in nine exercises that comprise both experimental data and numerical benchmarks encompassing wide range of fields from pure neutronics (exercise I-1: derivation of the multi-group microscopic cross-section libraries; exercise I-2: derivation of the few-group macroscopic cross-section libraries; exercise I-3: criticality stand-alone neutronic calculations with confidence bounds) to coupled phenomena in a core behavior (exercise II-1: fuel thermal properties relevant for transient performance; exercise II-2: neutron kinetics stand-alone performance; exercise II-3: thermal-hydraulic fuel bundle performance) to a system phase (exercise III-1: coupled neutronic/thermalhydraulic core performance; exercise III-2: thermal-hydraulics system performance; exercise III-3: coupled neutronic kinetics thermal-hydraulic core/thermal-hydraulic system performance).

The IRSN has the main task to support with technical assistance the French Safety Authority. For being able to provide effective assistance, it is necessary to always be at state-of-art of scientific knowledge and to anticipate the potential needs of tomorrow's expertise. For this reason, aiming to increase competence and to estimate the robustness of one or other methodologies applied in the uncertainty propagation domain, the IRSN took part in UAM-LWR activities with contributions in the benchmark.

In this paper, we show the results of uncertainty propagation on a rod ejection accident, on a whole core; exercise III-I-2c [4]. The core is in a Hot Zero Power state, with $18 \mathrm{GWd} / \mathrm{tHM}$ mean exposure. As specified in the benchmark (based on licensing Three-Mile-Island Unit 1 power upgrade calculations), the nature of the uncertainties is thermal-hydraulic (TH) and neutronic. The computations were executed with a part of the 3D computational IRSN chain HEMERA [5]: the coupling of the neutronic core code CRONOS [6] and the thermal-hydraulic core code FLICA4 [7]. CRONOS computes the power distribution and it works with two energy groups, 4 nodes by the assembly. FLICA4 computes the moderator density distribution and it has one channel by the assembly. As in our first UAM exercise (numerical rod ejection, [8]), we used the cross sections standard deviations supplied by the UAM team [9] and applied (in percentage, following a Gaussian probability law and uniformly to the entire core) to the core cross sections. The TH uncertainties applied to FLICA are: initial power, flow rate, pressure, and inlet temperature. To these uncertainties, defined by the benchmark, we have added the nuclear energy fraction directly released in the moderator, with $1 \%$ variability, and the fuel to cladding heat transfer coefficient, with $20 \%$ variability: we both gave a uniform probability law (we estimated these values as consistent with those provided by the benchmark).. To increase the power peak of the original benchmark proposition, we executed a supplementary rod ejection with a bigger reactivity injection in the core $(0.78 \$$ the first case, $1.14 \$$ the second case). Simulations last 1 second.

The UAM-LWR exercise presumes propagating uncertainties along the entire core transient. The list of uncertainties to be propagated included as few-groups cross sections (XS) uncertainties and ones of thermal-hydraulic parameters. Applied in the studies brute-force sampling methodology, i.e. numerous transient computations where XS and thermal-hydraulic parameters were varied within given bounds of uncertainties, allowed estimate standard deviations of outputs including peak-of-power, deposit energy and time of peak. Probability Density Function was postulated to be Gaussian one taken directly from UAM-LWR specification. In the following, after the benchmark description (section 2), we present some results (section 3). Section 4 is dedicated to some sensitivities analysis and section 5 contains some comments about all the results presented in this paper.

\section{BENCHMARK DESCRIPTION}

The rod ejection is executed in a full core [4], Figure 1, composed of 177 PWR assemblies, surrounded by a reflector. Side, top, and bottom reflectors are identical. The reflector model is a 1D model: fuel (21.6 $\mathrm{cm})$, water gap $(0.21 \mathrm{~cm})$, stainless steel shroud $(1.91 \mathrm{~cm})$ and water $(19.521 \mathrm{~cm})$. The active core height is $365.76 \mathrm{~cm}$, side and axial reflectors are $21 \mathrm{~cm}$ tall. The fuel rod pitch is $1.443 \mathrm{~cm}$ and the fuel + clad 
diameter is $1.092 \mathrm{~cm}$. The assemblies have $15 \times 15$ pins: seven different types of assemblies are charged in the core [4]: with a U235 enrichment going from 4 to $5 \% \mathrm{w} / \mathrm{o}$ and from 0 to 8 Gadolinium pins. The control rods are composed by AIC (Silver-Indium-Cadmium).

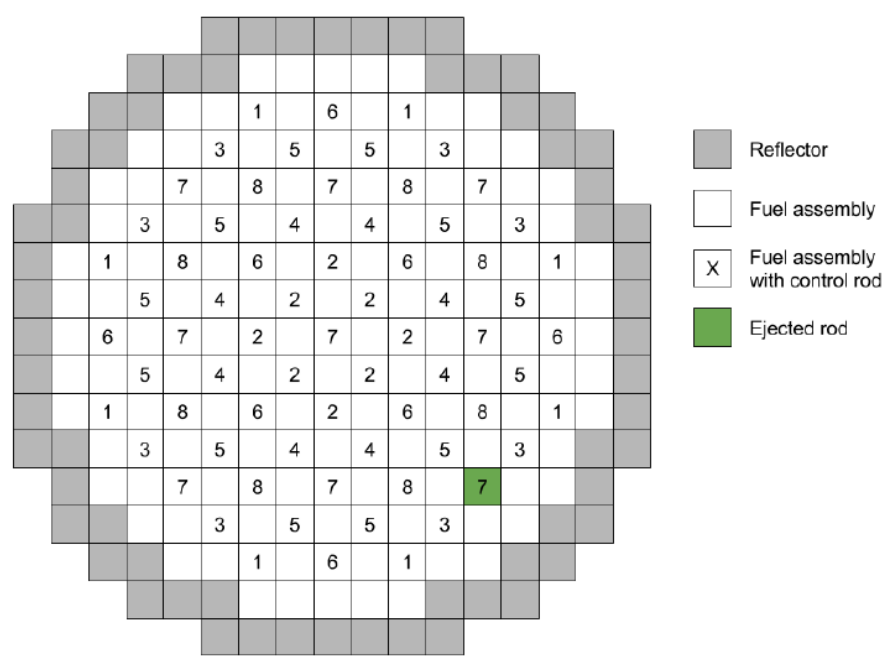

Figure 1: TMI Core (Hou et al, 2019)

The core is in a BOC (Beginning Of Cycle) state, with an assembly based exposure map imposed by the benchmark. The state of the core is summarized in Table 1:

Table I: Core Steady State

\begin{tabular}{|l|c|}
\hline Power & $2771.9 \mathrm{~kW}(0.01 \%$ Nominal Power $)$ \\
\hline Average Fuel Temperature & $551^{\circ} \mathrm{K}$ \\
\hline Average Moderator Temperature & $551^{\circ} \mathrm{K}$ \\
\hline Average Exposure & $18.08 \mathrm{GWd} / \mathrm{tHM}$ \\
\hline Control Rods Groups 1-4 & Completely withdrawn \\
\hline Control Rods Groups 5 -7 & Fully Inserted \\
\hline Control Rod Group 8 & $70 \%$ inserted \\
\hline Core Inlet Pressure & $15.36 \mathrm{MPa}$ \\
\hline Core Flow Rate & $16546.07 \mathrm{Kg} / \mathrm{s}$ \\
\hline
\end{tabular}

The rod 7 (Figure 1), fully inserted, is ejected at time zero of the transient with a constant rate of 2380.8 $\mathrm{cm} / \mathrm{s}$.

The benchmark specifications presume computing of the means value and the standard deviations to be associated with all given transients. The deviations had to be calculated perturbing cross sections in the frame of the postulated uncertainties of initial nuclear data (see details below).

\section{BENCHMARK EXECUTION AND RESULTS}

Below one could find a quick description of XS variation in the sampling calculations and some noted on the computational process with the major outputs. 


\subsection{Uncertainties Treatment}

The goal of the benchmark is to study the uncertainties propagation, i. e., to compute a time-dependent mean power and its associated time-dependent standard deviation. Two types of uncertainties has to be propagated: neutronic (associated with the cross sections and to the variations of the kinetic parameter) and thermal-hydraulic (associated with the core boundary conditions). To the already specified thermalhydraulic uncertainties, we have added two supplementary uncertainties (the fuel to cladding heat transfer coefficient and the nuclear energy fraction directly released in the moderator) and not used two: the power distribution and the cladding to coolant heat transfer coefficient (computed by FLICA and not modifiable).

The cross sections and the kinetic parameters were modified according to a normal Probability Distribution Function (PDF) with a standard deviation supplied by the UAM team [9]. Figure 2 shows the standard deviation (in \% of the cross section or kinetic parameter) we used:

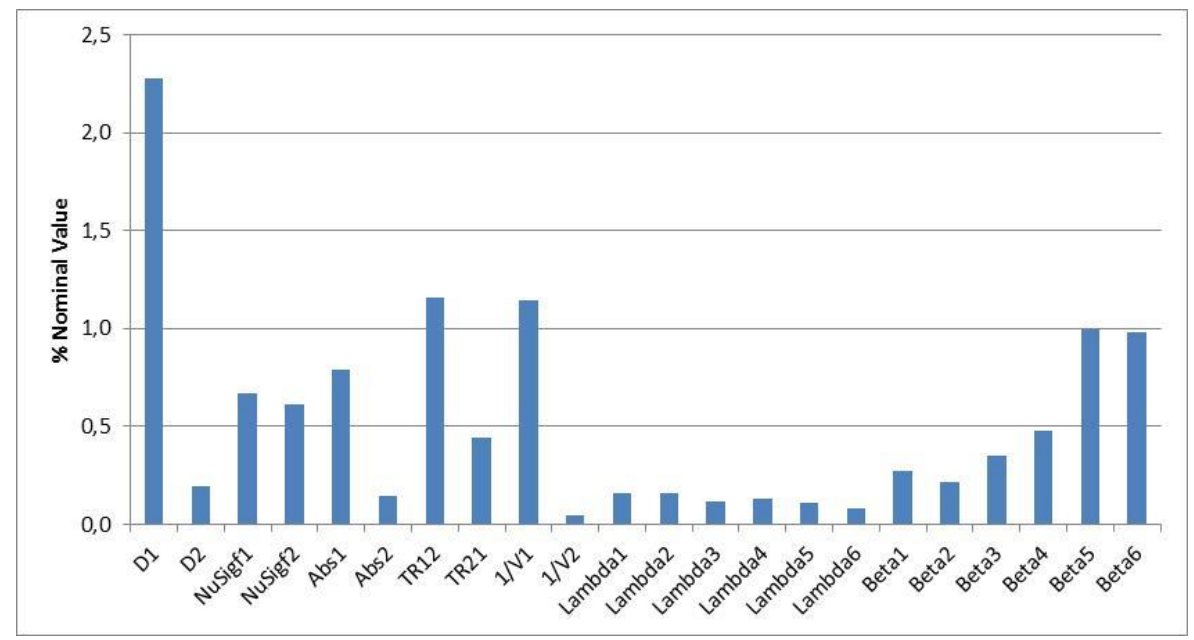

Figure 2: Cross Sections Standard Deviation at 18 GWd/tHM (see Nomenclature)

In the case of thermal-hydraulic parameters, we used the assumed maximum variations differently following if the PDF uniform or normal. In the case of the uniform PDF, the parameter was randomly modified between the minimum and the maximum variation. For a normal PDF, the maximum variation was interpreted as 3 times the standard deviation.

Table II describes the PDF and the maximum variations: 
Table II. TH Uncertainties List

\begin{tabular}{|c|c|c|}
\hline Parameter & PDF & Maximum Variations \\
\hline $\begin{array}{c}\text { Fuel to Cladding Heat Transfer } \\
\text { Coefficient }\end{array}$ & Uniform & $+/-20.0 \%$ \\
\hline $\begin{array}{c}\text { Nuclear Energy Fraction Released } \\
\text { in the Moderator }\end{array}$ & Uniform & $+/-1.0 \%$ \\
\hline Inlet Fluid Temperature & Uniform & $+/-1.0 \%$ \\
\hline System Pressure & Normal & $+/-1.0 \%$ \\
\hline Flow rate & Normal & $+/-1.5 \%$ \\
\hline Power & Normal & $+/-1.0 \%$ \\
\hline $\begin{array}{c}\text { Cross Sections and Kinetic } \\
\text { parameters }\end{array}$ & Normal & $\begin{array}{c}3 \text { times the Standard } \\
\text { Deviation (Figure 2) }\end{array}$ \\
\hline
\end{tabular}

It should be noted that all the relative variations (neutronics and thermal-hydraulics) were defined at the beginning of the transient and their values have been kept constant for the continuation.

\subsection{Benchmark Execution}

All the computations were executed with the branch 'core' of HEMERA [5]: the coupling of the neutronic core code CRONOS [6] and the thermal-hydraulic core code FLICA [7]. The coupling is explicit: CRONOS gets the fuel temperature and moderator density distributions from FLICA, computes the power distribution and sends it to FLICA. For a rod ejection, the time step used is $0.001 \mathrm{sec}$. The core modeling of CRONOS is: 2 energy groups (thermal group $<0.625 \mathrm{eV}$, fast group $>0.625 \mathrm{eV}$ ), 4 meshes by the assembly and 26 axial meshes (imposed value) and one channel by the assembly for FLICA.

The initial steady-state is critical, with the rod fully inserted. We made two series of calculations: the first, injecting in the core a reactivity of $0.78 \$$ (as suggested by the benchmark), the second injecting $1.14 \$$ (simply modifying the rod ejection distance from the bottom of the core). The reason is to have a stronger power peak.

The sequence of the calculations is, for each transient:

- Neutronics and thermal-hydraulic parameters have been computed starting from their nominal values and according to their probability distribution. Searching for the critical boron;

- The transient starts at zero time. The rod was ejected in less than 0.1 seconds (ejection speed is imposed by the benchmark [4], therefore the ejection time depends on the initial position of the rod) while the computation lasts one second.

The time-dependent mean power is obtained by doing the mean of all power curves (about 300 for every calculation).

\subsection{Results and comments}

Figure 3 shows the mean power (MW) and the standard deviation (in percentage of the mean power) of the two calculations: the one with an injected reactivity of $0.78 \$$ (A, $20 \mathrm{MW}$ after 1 second) and $1.14 \$$ (B, power peak of $1000 \mathrm{MW})$. 


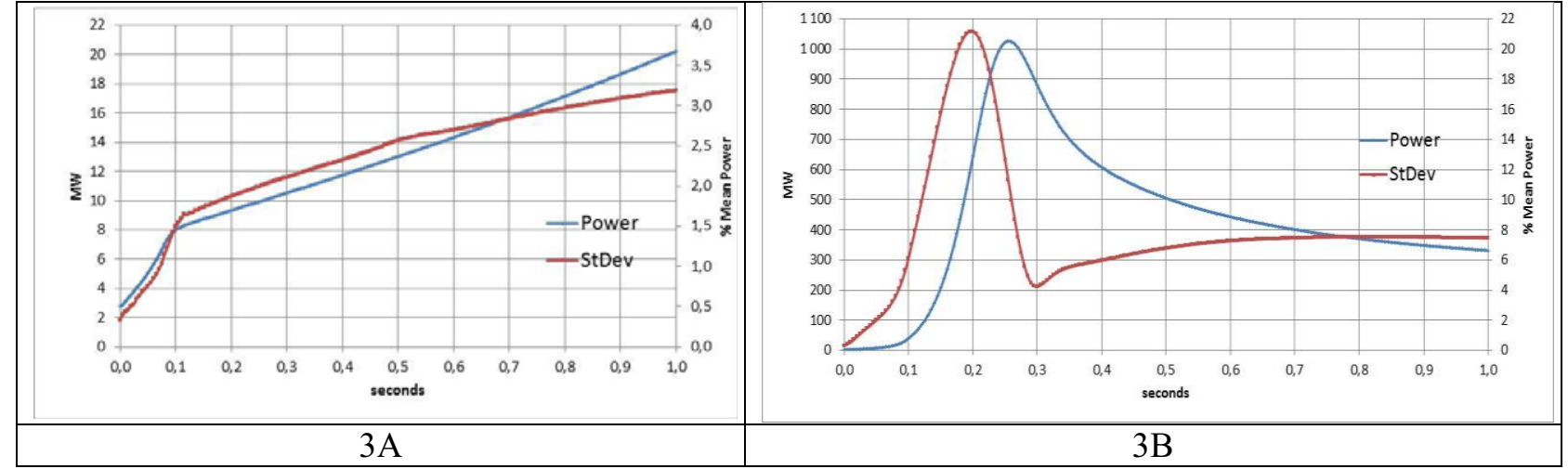

Figure 3: Mean Power (blue, MW) and Standard Deviation (red, \% of mean power)

In the case of $0.78 \$$ reactivity injected $(3 \mathrm{~A})$, the standard deviation, in percentage, follows closely the mean power curve. In the case of $1.14 \$$ reactivity injected (3B), the standard deviation peak is shifted of 0.5 seconds, compared to the power peak time. In our previous mini-core case [8], purely neutronic without feedback, we always found curves very similar to the $3 \mathrm{~A}$ ones. We can understand this similarity because, in the mini-core, there was no feedback; in this case, the power grows slowly and the feedback is very small.

Figure 4 illustrates the ratio between the time-dependent partial variances (only due to neutronic parameters and only due to thermal-hydraulic parameters) and the time-dependent total variance. The partial variances were obtained modifying, one at a time, the neutronic parameters set and the thermalhydraulic parameters set.

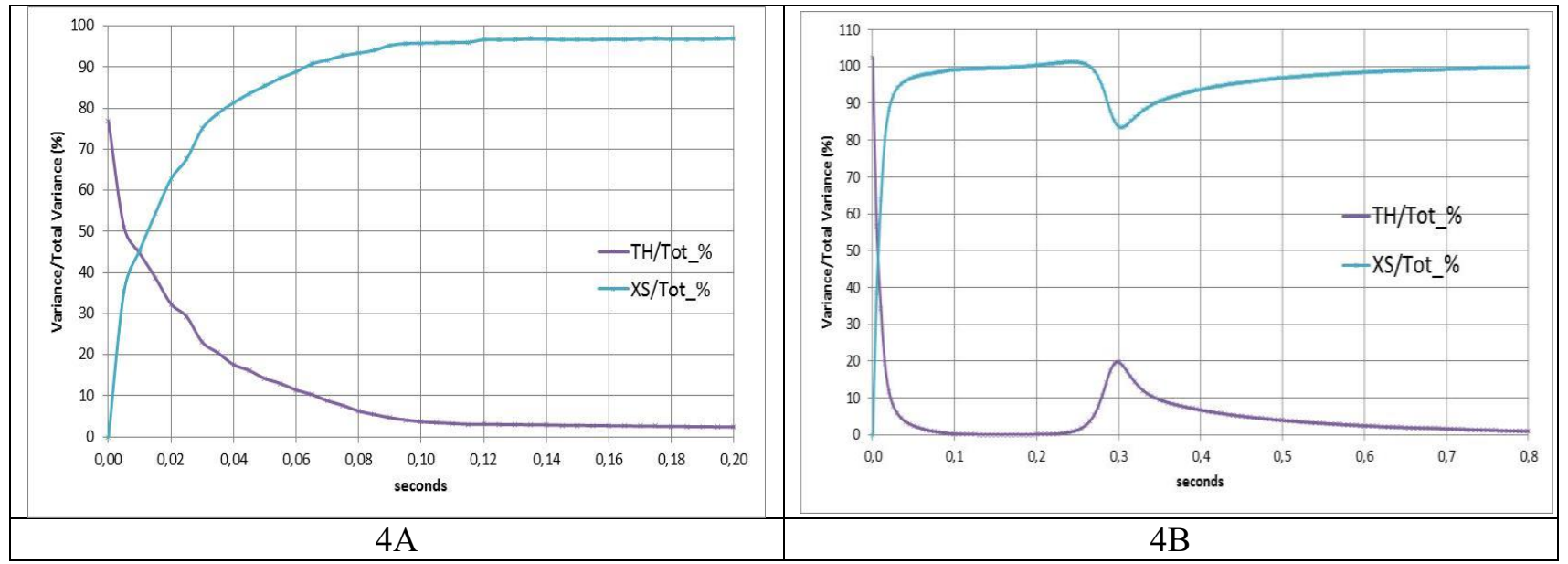

Figure 4: Partial Variances. 4A: 0.78\$ reactivity, 4B 1.14\$ reactivity (after $0.16 s$ and $0.8 s$ the curves are constant)

With a reactivity of $0.78 \$$, the importance of the thermal-hydraulic parameters decreases very quickly (practically, only the variation of the initial power is important) and, when the rod is out, $0.1 \mathrm{~s}$, only the neutronic parameters (the cross sections, actually) matter.

In the $1.14 \$$ case, the situation is a little different: at the transient beginning neutronic parameters become dominant but quite late, around 0.3 seconds, a thermal-hydraulic peak appears and this peak coincides 
with the minimum (in \%) of the total standard deviation curve (Figure 3B). A deeper analysis shows that, between the thermal-hydraulics parameters, the fraction of nuclear energy directly released in the moderator is the only cause of this peak. The explication of this peak is still in progress.

Between the neutronic parameters, only the fast energy group is responsible for power peak variance. Figure 5 shows the largest contributions (case $1.14 \$$, at $0.225 \mathrm{sec}$, power peak, and at $0.220 \mathrm{sec}$., max standard deviation) between all the neutronic parameters and, for comparison, the contribution of the nuclear energy fraction released in the moderator.

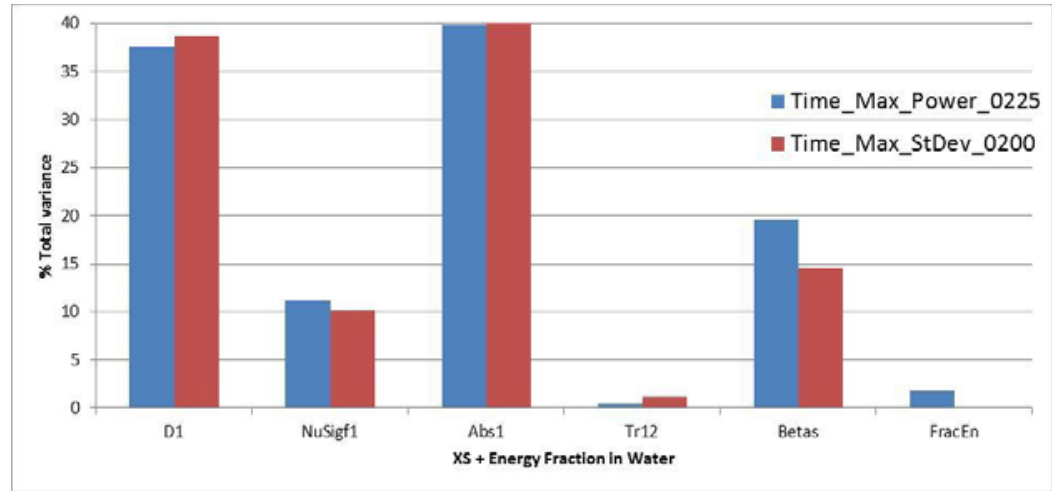

Figure 5: Cross Sections Variances on Total Variance (Power Peak)

Figure 5 demonstrates the similar findings as in a case of mini-core [8]. The biggest contributors are the D1 and Abs1 (1st groups of diffusion and absorption). The NuSigfl and Beta ( $1^{\text {st }}$ group production and delay neutron effective fraction) are also notable. Others, including $\operatorname{Tr} 12$ (moderation from the $1^{\text {st }}$ to the $2^{\text {nd }}$ group) despite on significant standard deviation (Figure 2) has a little contribution (see [8]).

\section{CONCLUSIONS}

The paper presents one exercise of the OECD-NEA UAM-LWR project, the exercise III-I-2c that was aimed at uncertainty propagation through a transient caused by a fast (full ejection in 0.1 seconds) ejection of the control rod in a full PWR core.

Two cases were studied: the first one (suggested by the benchmark) with a reactivity insertion of $0.78 \$$, and the next one (added by authors) with reactivity increased up to $1.14 \$$. Authors simulated the transient using coupled CRONOS (core neutronics) and FLICA4 (core thermal-hydraulics) codes modeling full core behavior. In both cases, the uncertainties due to nuclear data and thermal-hydraulics parameters have been propagated in a form of Gaussian probability distribution functions outlining standard deviations for peak-of-power, energy release and so on.

In the case of $0.78 \$$ the power increase is quite small and the impact due to feedback was small as well. The time-dependent standard deviation curve (in \% of the mean power) closely follows the timedependent mean power curve. This behavior is very similar to what we found in a previous UAM exercise [8]: a rod ejection in a mini-core with fuel and moderator temperature imposed. It is easy to show that, the common point between these two exercises that was is the missing, or weakness, of the reactivity feedback impacts, making growing uncertainty of power faster than of power itself. At any rate, one can see that total transient uncertainties are driven mainly by the uncertainties of used nuclear data. 
The observations after the second case of $1.14 \$$ results are different a little bit. The peak-of-power is achieved around $1000 \mathrm{MW}$ where an impact, due to reactivity feedback, becomes substantial. The timedependent standard deviation curve (\% of mean power) increases with the mean power but it has a maximum before the peak itself. Moreover, even if the neutronic uncertainties always explain most of the total mean power variance, the fraction of nuclear energy directly deposed in the moderator seems to play a not negligible role, explaining $20 \%$ of the total variance around 0.3 seconds (but only about $2 \%$ of the peak power variance, Figure 5). At this time, the rod is already completely out since 0.2 seconds. The explication of this result is still in progress.

In both cases, the most important contributors to the total variance are the fast-group cross sections (Figure 5 for the $1.14 \$$ case. For the $0.78 \$$ case, it is the same); in particular, the fast diffusion coefficient and the fast absorption. This result, analogous to the mini-core one [8], seems to confirm the relevance of the fast group cross sections variability on the mean power variability. However, a question could arise about the cross sections standard deviations magnitude: their predominance on total variance is real or due to an uncertainties over-estimation? This question is still open.

\section{NOMENCLATURE}

$\mathrm{D} 1,2$ = fast and thermal diffusion coefficients, NuSigf1,2 = fast and thermal production cross sections; Abs1,2 = fast and thermal absorption cross sections; $\operatorname{Tr} 12=$ scattering from group 1 to 2 ; $\operatorname{Tr} 21=$ scattering from group 2 to $1 ; 1 / \mathrm{V} 1,2=$ inverse of the neutron velocity for the fast and thermal group; Lambda1..6 and Beta1..6 = decay constant and delay neutrons fractions by precursors family.

\section{ACKNOWLEDGMENTS}

Authors appreciate all the UAM-LWR expert group members, especially its leaders Tomasz Kozlowski and Kostadin Ivanov for fruitful scientific discussions and support of the presented studies.

\section{REFERENCES}

1. F. D'Auria, S. Langebuch, 'Technology relevance of the "Uncertainty Analysis Modelling” for Nuclear Reactor Safety', NEA/NSC/DOC(2007)15, Nov. 2007

2. K. Ivanov et al.; 'Benchmarks for uncertainty analysis in modelling (UAM) for the design, operation and safety analysis of LWRs. Volume I: Specification and Support Data for Neutronics Cases (Phase I)', NEA/NSC/DOC(2013)7, Nov. 2013

3. J. Hou et al., 'Benchmark for Uncertainty Analysis in Modelling (UAM) for Design, Operation and Safety Analysis of LWR, Phase II', NEA/NSC/DOC(2014), April 2017

4. J. Hou et al., 'Benchmark for Uncertainty Analysis in Modelling (UAM) for Design, Operation and Safety Analysis of LWR, Phase III', NEA/NSC/DOC(2019), April 2019

5. F. Dubois, B. Normand, A. Sargeni, "An example of Neutronic penalizations in reactivity Transient Analysis Using 3D Coupled Chain HEMERA", Physor 2012, April 15-20, 2012, Knoxville, US-TN

6. J.J. Lautard, S. Loubière, C. Magaud,"CRONOS, A Modular Computational System for Neutronics Core Calculations", IAEA Specialists Meeting, France, September 1990

7. I. Toumi et al.,"FLICA4: a Three Dimensional Two-phase Flow Computer Code with Advanced Numerical Methods for Nuclear Applications", Nuclear Engineering and Design, 200 (2000)

8. A. Sargeni, F. Fouet, E. Ivanov, P. Probst, "An IRSN contribution to the UAM project: mini-core, numerical rod ejection exercise", PHYSOR 2018, Cancun, Mexico, Avril 22-26, 2018.

9. https://www.oecd-nea.org/science/wprs/egrsltb/UAM/index.html 